\title{
Chinese Question Answering System Based on the Chinese FAQ
}

\author{
Xinlei Wang, Huijian Han \\ Shandong Provincial Key Laboratory of Digital Media, Shandong University of Finance and Economics, Jinan, Shandong, China
} (3052369013@qq.com), (while208@126.com)

\begin{abstract}
Sentence similarity computation is a key part of the Chinese FAQ system and the computation accuracy determine the quality of the system. Combining with question classification index, a new Chinese sentence similarity computing method based on Content Forward Network is proposed. The method combined concept semantic matching and the overall semantic question sentence similarity computation with question automatic classification to deal with the sentence similarity computing. Comparing with the traditional keyword-based and simple semantic question similarity computation method, Chinese frame semantic information makes the Chinese sentence similarity calculation accuracy improved obviously. The experimental results show that the method can effectively solve the intelligent matching problem in Chinese question answering system.
\end{abstract}

Keywords—Sentence similarity; Chinese Frame Network; Frame element; Question classification; Question answering system

\section{基于汉语框架网的中文 FAQ 问答系统}

\author{
王新否 韩慧健 \\ 山东财经大学 山东省数字媒体技术重点实验室, 济南, 山东, 中国
}

\begin{abstract}
摘 要 问句相似度计算是中文 FAQ 问答系统的关键部分, 其计算精度决定系统的质量。结合问句分类索引提出了基于汉语框 架网的问句相似度计算新方法。该方法在问句自动分类匹配基础上, 结合概念语义与问句整体语义进行问句相似度计算, 与传统基于 关键词和单纯语义的问句相似度计算方法相比, 汉语框架语义信息的引入使得中文问句相似度计算精度得到明显提高。实验结果表明 该方法能够有效解决中文问答系统的中问题智能匹配等问题。
\end{abstract}

关键词 问句相似度, 汉语框架网, 框架元素, 问句分类, 问答系统

\section{1. 引言}

问答技术旨在令计算机能够回答用户用自然语言提问 的问句 (尤其是在语音交互情况下), 对问答技术的研究一 直是信息检索和自然语言处理领1域的重要研究内容, 其中 FAQ（Frequently Asked Question）问答系统在现实生活中 已经被广泛应用。基于 FAQ 的问答系统是一种在已有的“问 题-答案” 对集合中找到与用户咨询问题匹配的问句, 并将 其对应的答案反馈给用户的问答式检索系统 [1]。该系统将 人们常咨询的问题和相关答案进行关联保存, 对于用户输 入的咨询问句, 首先在常用问题库中进行查找, 如果和以

国家自然科学基金重点项目 (60933008); 国家自然科学基金 (60903109, 60603077), 通讯作者: 韩慧健
往的查询记录相符, 则可直接将该问题对应的答案反馈给 用户, 而不需要经过问题分析、信息检索、答案抽取等重 新组织答案的复杂处理过程, 不仅能够提高系统的效 率, 同时还提高了答案的准确性。其中关键的问题是计算 用户咨询问句与 FAQ 问答系统问题库中问句的相似度, 同 时充分体现上下文咨询问句关系, 并把最佳结果反馈给用 户。

目前, FAQ 问答系统在国外研究相对比较成熟, 经典 的问答系统有麻省理工大学人工智能实验室的 Start[2]、密 歇根大学的 AnswerBus、IBM 基于统计的问答系统[3]等。

其中, Start 问答系统采用基于知识库和信息检索的混合模 式, 首先查找它自身知识库, 如查到反馈用户, 否则, 通 过搜索引擎检索反馈用户。在国内不少学者也在进行问答 
系统研究, 例如, 中科院计算所正在进行的大规模知识处 理科研项目 NKI (National Knowledge Infrastructure) 中的 一个具体应用就是 NKI 问答系统[4], 该系统向用户各领域 的知识服务, 支持自由式服务。

在目前这些已有的问答系统中, 只是从问句关键词概 念语义进行问句匹配忽略了句子的语法和整体语义等因 素, 使得提高语句相似度计算精度相对困难。本文研究汉 语框架网基础上, 提出了基于问句分类索引的问句相似度 计算方法, 并实现了基于该方法的山东财经大学招生 FAQ 问答系统。

\section{2. 汉语框架网}

汉语框架网 (Chinese FrameNet, CFN) 是一个以 Fillmore 的框架语义学 [5-6]为理论基础, 以加州大学伯克利 的 FrameNet []为参照和以汉语真实语料为依据供计算机使 用的汉语词汇语义知识库。其中, “框架” (Frame) 是跟一 些激活性语境 (Motivating Context) 相一致的机构化范畴 系统, 是存储人类认知经验中的图式化情景。框架中的参 与者称为框架元素, 分为核心框架元素和非核心框架元素。

$\mathrm{CFN}$ 数据库有框架库、句子库和词元库三部分组成。 框架库以框架为单位, 对词语进行分类描述, 明确给出框 架的定义和这些词语共有的语义角色即框架元素, 并描述 该框架和其他框架之间的语义关系; 句子库包含带有框架 语义标注信息的句子, 即按照框架库所提供的框架和框架 元素类型, 标注句子的框架语义信息和句法信息; 词元库 记录了词元的语义搭配模式和框架元素的句法实现方式, 覆盖认知、科普、旅游、教育和法律等多个领域。

CFN 提供了良好的问句语义信息, 为句子的相似度计 算提供丰富的语义资源, 能有效提高问句的检索效率。

\section{3. 系统设计与实现}

系统主要包括问句预处理模块、候选问题集的建立、 问句相似度计算模块和 FAQ 更新模块, 具体系统流程图可 见图 1。

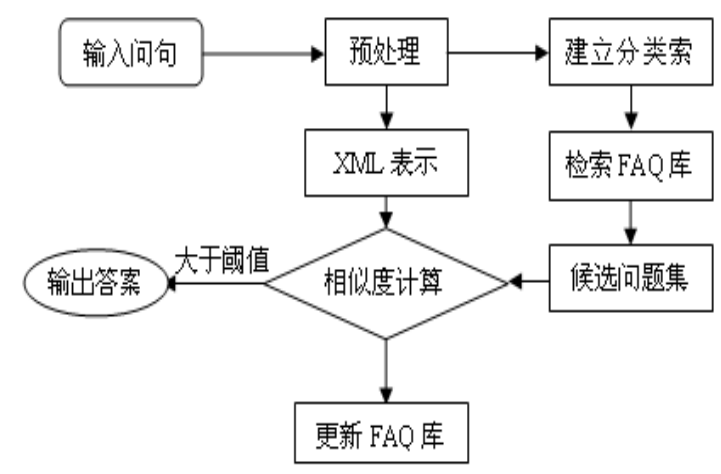

图 1 基于框架语义网的问答系统流程

\section{1 问句预处理}

问句预处理主要包括: 分词、词性标注、去停用词和 语义标注。

系统利用中国科学技术学院计算技术研究所研究的分 词组件 ICTCLAS[7]对问句进行分词和词性标注后, 将去除 停用词剩余的词作为关键词。解决关键词表示问句中的歧 义和指代关系等问题, 对问句进行 CFN 语义标注。根据 CFN 标注获取问句的主题、问点、谓词 (目标词)、疑问词 等信息, 利用同义词处理和领域概念逼近等算法, 形成规 范问句向量。为了保证 CFN 语义标注的准确性, 还需要建 立领域词汇词性库, 增加语义关系充实问句语料数据库。

如: “山东财经大学 2012 年招收多少本科生?”经过 分词、词性标注以及 CFN 语义标注后为 (其中山东财经大 学为本体领域词汇):

<ent-np-subj 山东财经大学 $/ n><$ time-tp-adva 2012 年 $/ \mathrm{t}>\langle\mathrm{tgt}=$ 招 招收/v $>\langle$ value-np-obj 多少/r 本科生/n>

\section{2 候选问题集的建立}

建立候选问题集的目的是缩小查找范围, 是后续的相 似度计算等较复杂的处理过程都在范围相对较小的候选集 内进行。为了克服传统基于关键词索引的问句重复性较高 问题, 本系统首先对用户问句进行归类处理, 将 FAQ 库中 属于同一归类的问句作为候选问题集。假设用户输入问句 属于问题分类体系中的, 则问句 的候选问题集为 FAQ 库 中问句分类 问句的集合。

为了能够快速地查找到问句候选问题集, 系统设计了 如图 1 所示数据结构。

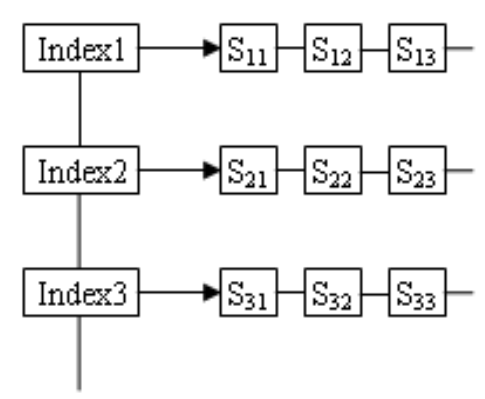

图 2 分类索引存储结构

建立分类索引的关键是对 FAQ 库中的问句进行分类, 系统参照哈尔滨工业大学信息检索研究室的中文分类体系 [8], 结合招生咨询系统的特点, 利用文献 [9]中陈述句的 CFN 标注规范, 对招生咨询问句进行 CFN 标注, 根据问句 疑问意向提取问句的分类特征[10]对招生领域的 1500 条常 
见问句进行分类处理, 将问句库中所有问句分为 7 类, 具 体分类统计见表 1 。

表 1 问题分类统计

\begin{tabular}{|c|c|c|}
\hline 大类 (Coarse) & 数量 & 百分比 \\
\hline 人物 (HUM) & 25 & $1.67 \%$ \\
\hline 地点 (LOC) & 59 & $3.93 \%$ \\
\hline 数字 (NUM) & 201 & $13.4 \%$ \\
\hline 时间 (TIME) & 91 & $6.07 \%$ \\
\hline (招生领域) 实体 & 400 & $26.67 \%$ \\
\hline 描述 (DES) & 424 & $28.26 \%$ \\
\hline 未知 (Unknown) & 300 & $20 \%$ \\
\hline
\end{tabular}

\section{3 问句相似度计算}

对用户问句经过类别索引检索到候选问句集后，下一 步主要是从候选集中找出和用户问句最相似的一个问句。 所用方法是计算目标问句与候选问题集中每个问句之间的 相似度，对应的相似度最大的问句就是要找的问句。

在招生咨询领域, 问句的识别主要是依据句子中的疑 问词以及疑问意向词来对问句进行识别, 同时考虑到动词, 因为本体只是条目的关系必然是动词。而动词在 CFN 标注 中有相应的框架, 从而可以找到具有语义的重要信息。针 对招生咨询领域问句较短、语法简洁等特点, 本文采用基 于汉语框架网的空间模型和语义概念结合问句相似度计算 方法, 并将该方法与基于 TF-IDF 的问句相似度计算方法和 基于语义的问句相似度计算方法分别进行了比较。

咨询系统中问句一般比较简短, 只包含一个目标词 (动 词), 倘若问句中包含多个目标词时, 系统仅适用距离疑问 词最近的目标词所对应的 CFN 框架语义信息作为问句所在 框架。所以本系统只考虑问句一个目标词和基于这个目标 词的框架依存图[11], 本算法分计算两个目标词的相似度 $\mathrm{SW}$ 和计算两个问句对应框架依存图中框架元素的相似度 SG 两步进行。

\subsection{1 目标词的相似度}

目标词的相似度包括目标词所在的框架相似度 SW 和 两个目标词元相似度 SH 两部分。

(1) 目标词框架相似度

框架中包含核心框架元素和非核心框架元素两种, 核 心框架元素对问句相似度影响为:

$$
S C E= \begin{cases}\frac{h}{\max \left(h_{1}, h_{2}\right)} & h=\min \left(h_{1}, h_{2}\right) \\ \frac{h}{\min \left(h_{1}, h_{2}\right)} & h=\min \left(h_{1}, h_{2}\right)\end{cases}
$$

其中, $h_{1}, h_{2}$ 分别表示框架 $F_{1}$ 和 $F_{2}$ 中的核心元素个

数; $h$ 代表框架 $F_{1}$ 和 $F_{2}$ 中同名核心元素个数。

由于核心元素和非核心元素对于表征一个框架本质是 一样的, 只是贡献不一样大, 因此可以使用同样的方法计 算两个框架非核心元素相似度 $S N E$ 。

处于框架网中两个不同框架之间的语义距离对两个框 架相似度具有重要影响, 假设位于框架网中的两个连通的 框架 $F_{1}$ 和 $F_{2}$ 最短路径上的分支权重序列为 $e_{1}, e_{2}, \cdots, e_{n}$, 其中 $n$ 为最短路径上分支个数; $e_{i} \in\{W I, W T, W S, W C\}$, 分别代表框架网中继承关系、前后关系、父子关系和因果 关系对语义相似度的影响权重。则由框架网络中语义距离 对两个框架相似度影响为:

$$
S E F=\prod_{i=1}^{n} e_{i}
$$

因此，两个目标词框架相似度计算公式为:

$$
S F=\alpha^{*} S C E+\beta^{*} S N E+\gamma^{*} S E F
$$

其中, $\alpha, \beta, \gamma$ 为影响因子, 且满足 $\alpha+\beta+\gamma=1$ 。

（2）两个目标词元相似度

CFN 关注的焦点是框架而不是词元, 因此单纯基于 CFN 的相似度计算方法会产生词元之间聚合关系缺乏问 题。例如: 没有明确指出词元之间的语义距离和语义联系 (同义、反义、近义)。系统采用《同义词词林》中的词义 编码[12]计算词与词之间的相似度 $S C$, 然后重点利用 CFN 以场景描述语义的优势, 计算目标词所在框架的相似度。 最后把这两个相似度组合相加就是目标词的相似度。

根据以上步骤可得两个目标词相似度计算公式为:

$$
S W=t * S C+(1-t) * S F
$$

\subsection{2 框架元素相似度}

对于在两个不同句子中拥有相同框架元素名称的两个 框架元素来书, 它们的相似度是由它们所含词来决定的, 每个框架元素中所含元素为关键词集合, 因此两个框架元 素之间的相似度为其所对应的关键词集合之间的相似度。

假设两框架相同框架元素对应关键词集合为 WordSet1, WordSet2。针对两个关键词集合之间相似度不 
对称事实, 利用基于 《同义词词林》词义编码方法先计算 关键词集合 WordSet 1 对关键词集合WordSet 2 的相似度 $S_{1}$, 然后计算关键词集合 WordSet 2 对关键词集合 WordSet 1 的相 似度 $\mathrm{S}_{2}$, 最后求取平均值作为两个关键词集合的相似度 S(WordSet1, WordSet2)。

由于核心元素和非核心元素对问句相似度计算影响不 同, 其对应的关键词集合亦是如此。假设问句 $A, B$ 对应 依存图中有 $m$ 个相同的核心框架元素（核心依存项）和 $n$ 个相同的非核心框架元素 (非核心依存项), 因此问句 $A$, $B$ 所在依存图中框架元素的相似度为:

$$
S F N=\frac{\alpha \sum_{i=1}^{m} S\left(A_{i}, B_{i}\right)}{m}+\frac{\beta \sum_{j=1}^{n} S\left(A_{j}, B_{j}\right)}{n}
$$

其中, $\alpha, \beta$ 分别代表核心元素和非核心元素对相似度 的影响, $\alpha>\beta$ 且 $\alpha+\beta=1$ 。

因此, 问句 $A, B$ 相似度整体相似度为:

$$
\operatorname{Sim}(A, B)=\alpha * S W+(1-\alpha) S F N
$$

其中, $\alpha$ 为调节因子, 它通过实验数据选取最优值, 本文实验取值为 0.6 。

\subsection{FAQ 库的更新}

如果相似度都小于阈值, 则系统判定用户提出的问题 在 FAQ 问答库中没有匹配的问句, 系统会将用户问句进行 保存, 并发送至后台管理的待更新模块进行记录, 然后由 管理员进行回答, 并将问句和对应答案加入到 FAQ 库中, 进行 FAQ 的及时更新。

\section{4. 试验结果与分析}

为了更好地评价系统性能, 我们以山东财经大学 2012 年本科招生咨询为例, 根据中国人的咨询交流习惯, 挑选 300 个中文常见咨询语句, 用来测试系统召回率。本文根 据改进的召回率和准确率计算方法[13]对系统进行评测, 系 统设置的相似度阈值为 0.7 (长期实践得出阈值为 0.7 时系 统性能最稳定) 进行试验, 试验结果如表 1 所示。

表 2 实验结果

\begin{tabular}{|c|c|c|}
\hline 方法 & 准确率(\%) & 召回率(\%) \\
\hline 基于 TF-IDF 句子相似度计算方法 & 78.3 & 79.6 \\
\hline 基于分解向量空间与语义的计算方法 & 84.9 & 81.5 \\
\hline 基于本文方法 & 89.32 & 85.41 \\
\hline
\end{tabular}

\section{参考文献(References)}

[1] Jijkoun V, Pijke M. Retrieving Answers from Frequently Asked Questions Pages on the Web //Proc. of the 14th ACM Int'l Conf. on Information and Knowledge Management. [S. 1.]: ACM Press, 2005

[2] Boris Katz, Gregory Marton, Gary Borchardt, et al. The START Natural Language Question Answering System. [2006-12-16]. http://start.csail.mit.edu

[3] A Ittycheriah, S Roukos. IBM's Statistical Question Answering System //Proceedings of the TREC-11 Conference. Gaithersburg: NIST Special Publication, 2002:394-401

[4] Cungen Cao. NKI - hot spot of science and technology of the 21 st century. The computer world, 1998, 5(2):1-3

[5] Fillmore C J. Frame semantics and the nature of language //Annals of the New York Academy of Sciences: Conference on the Origin and Development of Language and Speech, 1976, 280:20-32

[6] Fillmore C J, Wooters C, Baker C F. Building a large lexical data bank which provides deep semantics //Proceedings of the 15th Pacific Asia Conference on Language, Information and Computation,HongKong,2001:3-26

[7] Huaping Zhang. Calculation of the Chinese academy of Chinese lexical analysis system. [2006-12-16].

[8] Jingguang Sun, Dongfeng Kui. Chinese automatic classification problem based on hownet. Journal of Chinese Information Processing, 2007, 21(1):90-95

[9] Liping You. CFN labeling manual (V1.1). 2006

[10] Ru Li, Xiaoxiang Song, Wenjing Wang. Chinese question classification framework based on Chinese network. Computer Engineering and Applications, 2009, 45(31):111-137

[11] Shuanghong Li, Ru Li, Lijun Zhong. Based on the chunk of semantic core word automatic identification research framework elements. Journal of Chinese Information Processing, 2010,24 (1):30-36

[12] Zheng Ye, Hongfei Lin, Zhihao Lin. The FAQ in Chinese question answering system based on question similarity. Computer Engineering and Applications, 2007, 43(9):161-163

[13] BURKE R D, HAMMOND K J, KULYUKIN V, et al. Question answering from frequently asked question files: ex-periences with the FAQ finder system.AI Magazine, 1997, 18(1):57-66. 